\title{
Nueva sección especial de REurEDC sobre investigación basada en el diseño de secuencias de enseñanza- aprendizaje
}

\author{
Jenaro Guisasola iD \\ Universidad del País Vasco (España).jenaro.guisasola@ebu.eus \\ José María Oliva iD \\ Editor de la revista. Universidad de Cádiz. (España).josemaria.oliva@uca.es
}

New section of ReurEDC: Design-Based research of Teaching-Learning Sequences

Para citar este artículo: Guisasola J., Oliva J.M. (2020) Nueva sección despecial de REurEDC sobre investigación basada en el diseño de secuencias de enseñanza. Revista Eureka sobre Enseñanza y Divulgación de las Ciencias 17(3), 3001. doi: 10.25267/Rev_Eureka_ensen_divulg_cienc.2020.v17.i3.3001

La investigación basada en el diseño de secuencias de enseñanza surge del reconocimiento de la complejidad de las intervenciones en el aula y la insatisfacción con las metodologías existentes para explorar los resultados de tales intervenciones. Estas preocupaciones no son nuevas y la aparición de propuestas alternativas y nuevas estructuras didácticas para el diseño de secuencias de enseñanza/aprendizaje es una consecuencia natural de las preocupaciones en el campo de investigación en Enseñanza de las Ciencias.

Las investigaciones basadas en el diseño se definen como el diseño, implementación, evaluación y rediseño de productos curriculares de mediana escala que cubren la enseñanza y el aprendizaje de un tema específico. Estos trabajos incluyen secuencias de enseñanza con el objetivo de mejorar el aprendizaje de los estudiantes de temas específicos a pequeña escala (por ejemplo, algunas sesiones de clase) o a media escala (una secuencia completa de sesiones de clase sobre uno o varios temas del programa) pero generalmente no abordan el nivel a gran escala de un programa completo en uno o varios cursos académicos (Psillos y Kariotoglou 2016).

Una característica distintiva de las investigaciones basadas en el diseño de secuencias de enseñanza es su carácter dual que implica investigación y desarrollo, apuntando a un fuerte vínculo entre la enseñanza y el aprendizaje de un tema en particular. En términos generales son investigaciones que tienen como objetivo una intervención en el aula o contexto educativo real, orientadas al proceso (se evita un análisis sólo "entrada-salida"), y se centran en comprender y mejorar las intervenciones (Sandoval y Bell 2004). Además, se genera un producto que incluye actividades de enseñanza-aprendizaje bien investigadas y adaptadas empíricamente al razonamiento de los estudiantes (Méheut y Psillos 2004).

Las investigaciones basadas en el diseño tienen el potencial de estrechar la brecha entre la práctica educativa y la teoría, ya que tienen como objetivo desarrollar teorías sobre el aprendizaje específico del dominio y los materiales educativos diseñados para apoyar este aprendizaje. Por lo tanto, más allá de su enfoque en la producción de innovaciones educativas para ser implementadas, la investigación basada en el diseño genera teorías "humildes", en el sentido de locales. (Kelley 2003). 
Si bien existe una tradición de investigación en estructuras didácticas centrada en el desarrollo de estudios concretos, hay pocas publicaciones sobre principios teóricos específicos y estrategias que guían las decisiones de diseño de los investigadores. Aún más escasos son los informes de cómo estos principios y estrategias influyen en las decisiones sobre el diseño real de actividades y problemas. Estos informes no suelen incluir tareas sobre cómo elegir la redacción adecuada para introducir actividades o formular preguntas que fomenten el pensamiento y el discurso productivo (Kortland y Klaassen 2010). La ausencia en las publicaciones de justificaciones detalladas sobre las decisiones de diseño, implementación y evaluación hace muy difícil un trabajo concertado de construcción sobre propuestas existentes y su evaluación. El resultado es una dispersión de propuestas que dificultan un avance efectivo en este campo.

En resumen, el objetivo de la nueva sección especial "Investigaciones de diseño" es publicar artículos de la comunidad de enseñanza de las ciencias que se dediquen al diseño, desarrollo y evaluación de secuencias de enseñanza. En particular, se promueven investigaciones que se centren en cómo, cuándo y por qué un diseño específico de materiales curriculares funciona en la práctica. Este perfil de artículos está en sintonía con una de las ideas manifestadas desde la revista en un editorial reciente (Oliva, 2020), como es reivindicar la necesidad de detalles sobre el contexto o escenario didáctico en el que se realizan las investigaciones sobre enseñanza de las ciencias, superando así la idea del diseño didáctico ensayado como caja negra en la que se conoce cómo llegan los estudiantes y qué resultados finales obtienen, pero no qué ha sucedido entre un momento y otro.

Sirva este editorial como invitación a los autores para enviar manuscritos a esta sección especial de la revista.

\section{Referencias}

Méheut M. y Psillos D. (2004) Teaching-Learning sequences: aims and tools for science. Guest Editors: Martine Méheut and Dimitris Psillos. International Journal of Science Education 26(5).

Kelley A. (2003) Theme issue: The role of design in educational research. Educational Researcher 32(1), 3-4.

Sandoval W. A. y Bell P. (2004) Design-based research methods for studying learning in context: Introduction. Educational psychologist 39(4), 199-201.

Psillos D. y Kariotoglou P. (2016) Iterative desing of teaching-learning sequences. Springer.

Kortland K. y Klaassen K. (2010) Designing theory-based teaching-learning sequences for science education. FSME, Utrecht.

Oliva J. M $\mathrm{M}^{\mathrm{a}}$ (2020) Sobre la importancia de contextualizar las investigaciones en didáctica delas ciencias. Revista Eureka sobre Enseñanza y Divulgación de las Ciencias 17(1), 1001. doi: 10.25267/Rev_Eureka_ensen_divulg_cienc.2020.v17.i1.1001 